\title{
Early increase of NOX2-derived oxidative stress in children: relationship with age
}

\author{
Lorenzo Loffredo', Pasquale Pignatelli', Francesco Martino², Roberto Carnevale', Simona Bartimoccia' ${ }^{1}$ Elisa Catasca',
} Chiara Colantoni ${ }^{2}$, Cristina Zanoni ${ }^{2}$, Ludovica Perri' and Francesco Violi ${ }^{1}$

BACKGROUND: Studies conducted in the prepubertal period showed that biomarkers of oxidative stress decreased with increasing age in normocholesterolemic children (NC), and, conversely, they are persistently high in hypercholesterolemic children (HC). Nicotinamide adenine dinucleotide phosphate (NADPH) oxidase is the most important cellular source of reactive oxygen species. No data have been reported concerning the behavior of age-related oxidative stress generated by NOX2, the catalytic subunit of NADPH oxidase, in children.

METHODS: We performed a cross-sectional study comparing oxidized low-density lipoprotein (ox-LDL), as a marker of oxidative stress, and NOX2 activity, as assessed by blood levels of soluble NOX2-derived peptide (sNOX2-dp), in a population of 250 children, including $125 \mathrm{NC}$ and $125 \mathrm{HC}$.

RESULTS: $\mathrm{HC}$ had higher sNOX2-dp $(25.8 \pm 16.1 \mathrm{pg} / \mathrm{ml})$ and ox-LDL $(23.6 \pm 14.4 \mathrm{U} / \mathrm{I})$ levels as compared with NC (15.4 \pm 10.1 and $11.6 \pm 6.4 \mathrm{pg} / \mathrm{ml}$, respectively; $P<0.001)$. Multiple linear regression analysis showed that Ox-LDL and total cholesterol were the only independent predictive variables associated with sNOX2-dp. In NC, ox-LDL and sNOX2-dp significantly decreased from the first to the second quintile of age. In $\mathrm{HC}$, ox-LDL and sNOX2-dp levels did not show significant differences among quintiles of age.

CONCLUSION: This study suggests that NOX2 contributes to generating ox-LDL during the early phase of life in NC and to the persistent high oxidative stress in the prepubertal period of $\mathrm{HC}$.

A therosclerosis represents the leading cause of death in Western countries (1). Although this pathologic process is a typical adult disease, it is already detectable in children with classic cardiovascular risk factors. Autopsy studies performed in children or youths with established risk factors demonstrated an association with the presence and extent of atherosclerotic lesions in the aorta and coronary arteries (2-4). Among the classic risk factors for atherosclerosis, hypercholesterolemia seems to be a major determinant of early atherosclerosis (2-4). Therefore, autopsy studies documented an association between cholesterol and the extent and severity of atherosclerosis in infants, children, and adolescents (2-4).
Early increase of cholesterol is also relevant in the progression of atherosclerosis in adults (5).

Oxidative stress plays a crucial role in the initiation and progression of atherosclerosis (6). In particular, an imbalance between superoxide and nitric oxide production could be responsible for endothelial dysfunction, the primum movens of the atherosclerotic process (7). Nicotinamide adenine dinucleotide phosphate (NADPH) activation has been recognized as the major reactive oxygen species (ROS) source (8). We have provided evidence of a major role for NADPH oxidase in modulating artery tone and generating oxidative stress in hypercholesterolemic children (HC) (9).

Studies conducted in healthy children showed that biomarkers of oxidative stress decreased with increasing age from 1 to $11 \mathrm{y}$ (10). In particular, during the prepubertal period, there is an age-related decrease of oxidative stress markers such as 8-hydroxy-2'deoxyguanosine levels (10), oxidized low-density lipoprotein (ox-LDL) (10), urinary $\mathrm{F}_{2}$-isoprostanes (11), and plasma levels of myeloperoxidase (10). Of note, such "physiologic" decline of oxidative stress is not detectable in HC (10), who are, therefore, exposed to the potential damage of oxidative stress even before the pubertal period.

No data have been reported concerning the age-related behavior of oxidative stress generated by NOX2, the catalytic subunit of NADPH oxidase, in healthy children or HC. The aim of this study was to analyze the role of NOX2 in normocholesterolemic children (NC) and HC during the prepubertal period.

\section{RESULTS}

Clinical characteristics of the population are reported in Table 1. Apart from serum cholesterol, no significant difference was found between the groups for BMI, systolic and diastolic blood pressure, fasting blood glucose, age, or gender (Table 1).

\section{Oxidative Stress Indexes According to Hypercholesterolemia} HC had higher ox-LDL and soluble NOX2-derived peptide (sNOX2-dp) levels as compared with NC (Table 1). No significant difference for oxidative stress indexes was found between males and females in $\mathrm{HC}$ and NC (Table 2). Bivariate

II Clinica Medica, Sapienza University, Rome, Italy; ${ }^{2}$ Center of Clinic Lipid Research, Department of Pediatrics, Sapienza University, Rome, Italy. Correspondence:

Lorenzo Loffredo (lorenzo.loffredo@uniroma1.it)

Received 26 July 2012; accepted 19 December 2012; advance online publication 10 April 2013. doi:10.1038/pr.2013.55 
analysis in the overall population showed that sNOX2-dp was significantly associated with ox-LDL $(r=0.523 ; P<0.001)$ (Figure 1a), total cholesterol (TC) $(r=0.333 ; P<0.001)$ (Figure 1b), LDL cholesterol $(r=0.298 ; P<0.001)$ (Figure 1c), and BMI $(r=0.185 ; P=0.003)$ (Figure 1d).

sNOX2-dp levels were significantly associated with ox-LDL in both the HC $(r=0.485 ; P<0.001)$ and NC $(r=0.291 ; P=$ 0.001) population (Figure 1a).

To establish the independent predictors of sNOX2-dp in the overall population, a multiple linear regression analysis, including the variables linearly associated with the dependent

Table 1. Clinical characteristics of healthy subjects and hypercholesterolemic children (HC)

\begin{tabular}{|c|c|c|c|}
\hline & NC & $\mathrm{HC}$ & \\
\hline & $n=125$ & $n=125$ & $P$ \\
\hline Age (y) & $8.3 \pm 3.2$ & $8.2 \pm 2.9$ & 0.671 \\
\hline Gender & $\begin{array}{l}62 \text { Males, } \\
63 \text { females }\end{array}$ & $\begin{array}{l}61 \text { Males, } \\
64 \text { females }\end{array}$ & 0.899 \\
\hline $\begin{array}{l}\text { Systolic blood } \\
\text { pressure }(\mathrm{mm} \mathrm{Hg})\end{array}$ & $106 \pm 9$ & $107 \pm 9$ & 0.505 \\
\hline $\begin{array}{l}\text { Diastolic blood } \\
\text { pressure }(\mathrm{mm} \mathrm{Hg})\end{array}$ & $68 \pm 8$ & $69 \pm 8$ & 0.764 \\
\hline $\begin{array}{l}\text { Total cholesterol } \\
(\mathrm{mg} / \mathrm{dl})\end{array}$ & $154.1 \pm 18.5$ & $217.7 \pm 42.0$ & $<0.001$ \\
\hline $\begin{array}{l}\text { LDL cholesterol } \\
(\mathrm{mg} / \mathrm{dl})\end{array}$ & $91.1 \pm 23.3$ & $148.9 \pm 42.8$ & $<0.001$ \\
\hline $\begin{array}{l}\text { HDL cholesterol } \\
(\mathrm{mg} / \mathrm{dl})\end{array}$ & $51.8 \pm 13.7$ & $53.7 \pm 23.3$ & 0.387 \\
\hline Triglycerides (mg/dl) & $63.5 \pm 32.5$ & $77.3 \pm 51.4$ & 0.012 \\
\hline $\begin{array}{l}\text { Fasting blood } \\
\text { glucose }(\mathrm{mg} / \mathrm{dl})\end{array}$ & $77 \pm 10$ & $78 \pm 10$ & 0.660 \\
\hline BMI & $19.3 \pm 4.7$ & $18.6 \pm 4.1$ & 0.203 \\
\hline ox-LDL (U/I) & $11.6 \pm 6.4$ & $23.6 \pm 14.4$ & $<0.001$ \\
\hline sNOX2-dp (pg/ml) & $15.4 \pm 10.1$ & $25.8 \pm 16.1$ & $<0.001$ \\
\hline
\end{tabular}

Bold values represent $P<0.05$.

HDL, high-density lipoprotein; LDL, low-density lipoprotein; NC, normocholesterolemic children; ox-LDL, oxidized LDL; sNOX2-dp, soluble NOX2-derived peptide. variable, was performed; ox-LDL (SE: 0.069; standardized coefficient $\beta: 0.460 ; P<0.001$ ) and TC (SE: 0.019 ; standardized coefficient $\beta: 0.169 ; P=0.005 ; R^{2}=33 \%$ ) were the only independent predictive variables associated with sNOX2-dp levels (Table 3).

\section{Age-Related Oxidative Stress Indexes}

To investigate the behavior of sNOX2-dp in children according to age distribution, we summarized the age of the overall population in five quintiles (Quintile (Q)1: $<6 \mathrm{y}, n=25$; Q2: 6-7 y, $n=25$; Q3: 8-9 y, $n=25$; Q4: 10-11 y, $n=25$; Q5: 12-13 $\mathrm{y}, n=25)$; clinical characteristics of each quintile in $\mathrm{HC}$ and NC are reported in Table 4.

In NC, TC, high-density lipoprotein cholesterol, and triglycerides did not show any significant differences among quintiles of age; by contrast, sNOX2-dp levels $(P=0.02)$ significantly decreased from the first to the second quintile of age (Figure 2). In HC, the lipid pattern parameters ox-LDL and sNOX2-dp did not show any significant differences among quintiles of age (Figure 2). ox-LDL and sNOX2-dp were significantly higher in $\mathrm{HC}$ as compared with $\mathrm{NC}(P<0.001)$ from the second to the fifth quintile of age (Figure 2 and Table 4 ).

\section{DISCUSSION}

The main result of the current study is that NOX2 activity is persistently elevated during the prepubertal period in HC subjects, indicating that hyperchosterolemia elicits an early oxidative burden via overactivation of NOX2.

NADPH oxidase constitutes the main source of superoxide in phagocytic and vascular cells (8). The NADPH oxidasederived ROS are involved in several physiological functions (aerobic metabolism, immunologic responses, cellular signaling, regulation of gene expression, and cell differentiation $(8,12-14))$ and pathologic (e.g., atherosclerosis, tumorigenesis, and neurodegenerative diseases $(8,12-14))$ processes. In vascular cells, the NADPH oxidases are implicated in the atherosclerotic process (13). Upregulation of NADPH expression seems to be involved also in the anatomic and functional changes of the arterial wall occurring in HC $(15,16)$. As compared with

Table 2. Clinical characteristics of healthy subjects and hypercholesterolemic children (HC)

\begin{tabular}{|c|c|c|c|c|c|c|}
\hline & \multicolumn{3}{|c|}{$\mathrm{NC}$} & \multicolumn{3}{|c|}{$\mathrm{HC}$} \\
\hline & 62 Males & $P$ & 63 Females & 61 Males & $P$ & 64 Females \\
\hline Systolic blood pressure $(\mathrm{mm} \mathrm{Hg})$ & $107 \pm 9$ & 0.398 & $106 \pm 9$ & $107 \pm 9$ & 0.286 & $108 \pm 7$ \\
\hline Total cholesterol (mg/dl) & $152.9 \pm 20.4$ & 0.473 & $155.3 \pm 16.6$ & $216.1 \pm 38.7$ & 0.674 & $219.3 \pm 45.2$ \\
\hline LDL cholesterol (mg/dl) & $89.2 \pm 21.6$ & 0.347 & $93.1 \pm 25.0$ & $147.5 \pm 41.7$ & 0.728 & $150.2 \pm 44.2$ \\
\hline Triglycerides (mg/dl) & $64.3 \pm 35.5$ & 0.786 & $62.7 \pm 29.5$ & $82.0 \pm 55.1$ & 0.319 & $72.8 \pm 47.4$ \\
\hline Fasting blood glucose (mg/dl) & $79 \pm 10$ & 0.167 & $77 \pm 10$ & $80 \pm 8$ & 0.703 & $79 \pm 9$ \\
\hline BMI & $19.4 \pm 4.2$ & 0.895 & $19.3 \pm 5.3$ & $19.1 \pm 4.1$ & 0.109 & $18.0 \pm 3.5$ \\
\hline ox-LDL (U/l) & $13.5 \pm 8.3$ & 0.408 & $12.3 \pm 8.2$ & $22.3 \pm 13.1$ & 0.692 & $24.0 \pm 15.7$ \\
\hline sNOX2-dp (pg/ml) & $16.1 \pm 10.9$ & 0.454 & $14.7 \pm 9.4$ & $25.1 \pm 15.5$ & 0.461 & $26.4 \pm 17.1$ \\
\hline
\end{tabular}

HDL, high-density lipoprotein; LDL, low-density lipoprotein; NC, normocholesterolemic children; ox-LDL, oxidized LDL; sNOX2-dp, soluble NOX2-derived peptide. 


\section{Articles | Loffredo et al.}
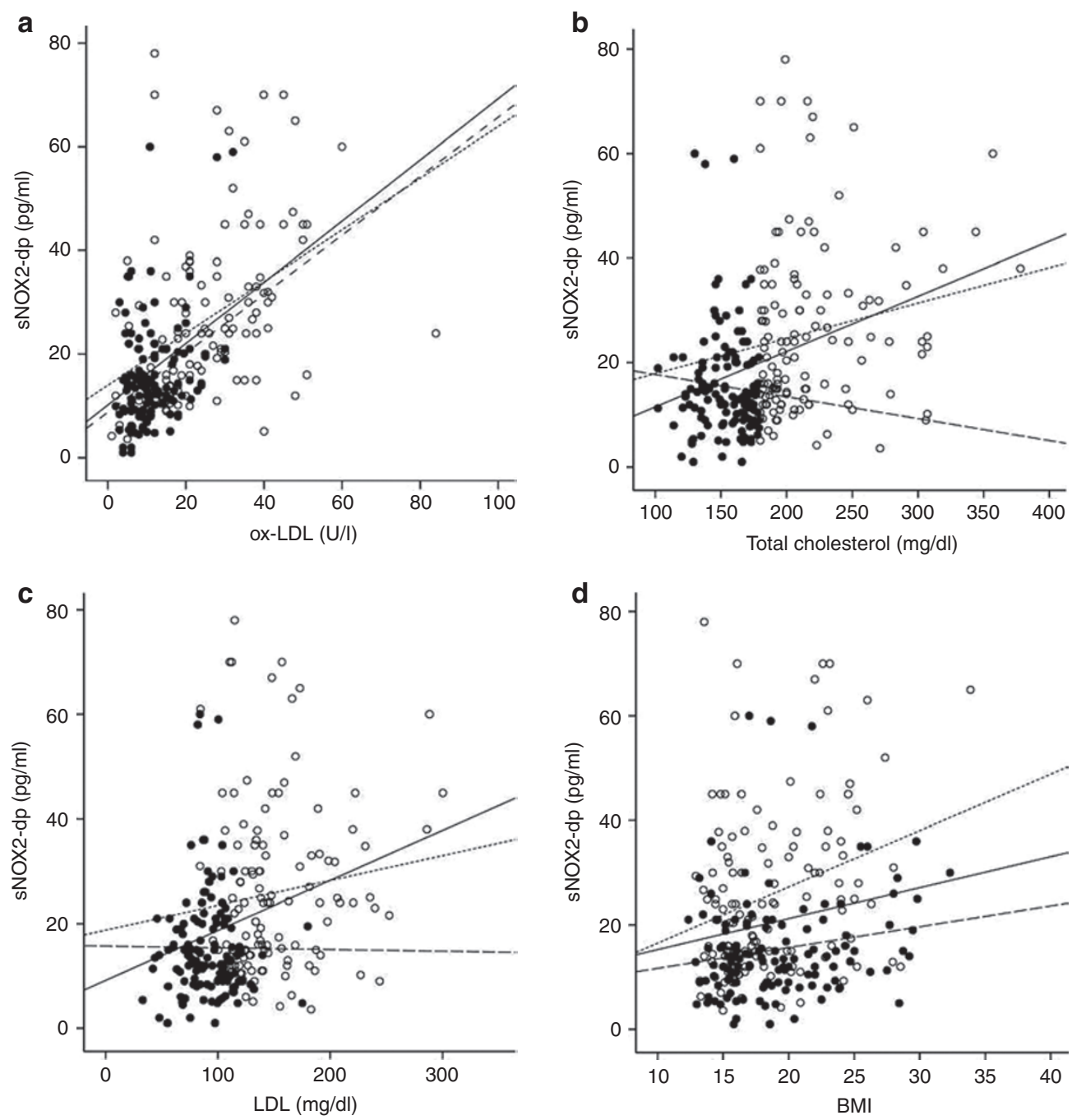

Figure 1. Linear regression analysis between (a) sNOX2-dp and ox-LDL, (b) total cholesterol, (c) LDL cholesterol, and (d) BMI. Black circles: NC group; white circles: HC group. HC, hypercholesterolemic children; NC, normocholesterolemic children; ox-LDL, oxidized low-density lipoprotein; sNOX2-dp, soluble NOX2-derived peptide.

Table 3. Multiple linear regression analysis

\begin{tabular}{lcccc}
\hline Model & $\begin{array}{c}95 \% \\
\text { Confidence } \\
\text { interval }\end{array}$ & $\begin{array}{c}\text { Unstandardized } \\
\text { coefficients }\end{array}$ & $\begin{array}{c}\text { Standardized } \\
\text { coefficients }\end{array}$ & \\
\cline { 3 - 4 } ox-LDL & $0.368-0.624$ & 0.069 & $\beta$ & $P$ \\
\hline $\begin{array}{l}\text { Total } \\
\text { cholesterol }\end{array}$ & $0.018-0.090$ & 0.019 & 0.460 & 0.000 \\
\hline
\end{tabular}

Dependent variable: sNOX2-dp.

ox-LDL, oxidized low-density lipoprotein

NC, we previously observed an overexpression of platelet gp91 (phox), reduced flow-mediated dilation, and enhanced intima media thickness in $\mathrm{HC}$ and, conversely, higher flow-mediated dilation and lower intima media thickness and platelet gp91 (phox) in children with hereditary deficiency of NOX2 (15).

Although this finding is consistent with our previous study demonstrating NOX2 upregulation in children with atherosclerotic risk factors (17), it underscores the fact that the burden of oxidative stress-related atherosclerotic risk appears in the early phase of life and is dependent on NOX2 regulation.

Therefore, data on the behavior of oxidative stress in childhood consistently showed an enhanced oxidative stress in the first years of life, which has been suggested to reflect a response to higher infection rate (18) or the more rapid basal metabolic rate occurring at this age (17); subsequently, oxidative stress tends to gradually decline until the age of $11 \mathrm{y}$ (19). It is likely that more than one pathway generating ROS is implicated in enhancing oxidative stress during the first years of life. Consistent with this hypothesis is the previous demonstration of myeloperoxidase upregulation and the results of the current study showing NOX2 upregulation.

We also examined if during the prepubertal period, children with hypercholesterolemia show a different behavior of NOX2 and its impact with oxidative stress. This issue is of potential interest because $\mathrm{HC}$ have early manifestations of atherosclerotic disease that may be related to prolonged exposure to oxidative stress. In this study, we found that, as compared with 
Table 4. Clinical characteristics of healthy subjects and hypercholesterolemic children (HC) according to quintile

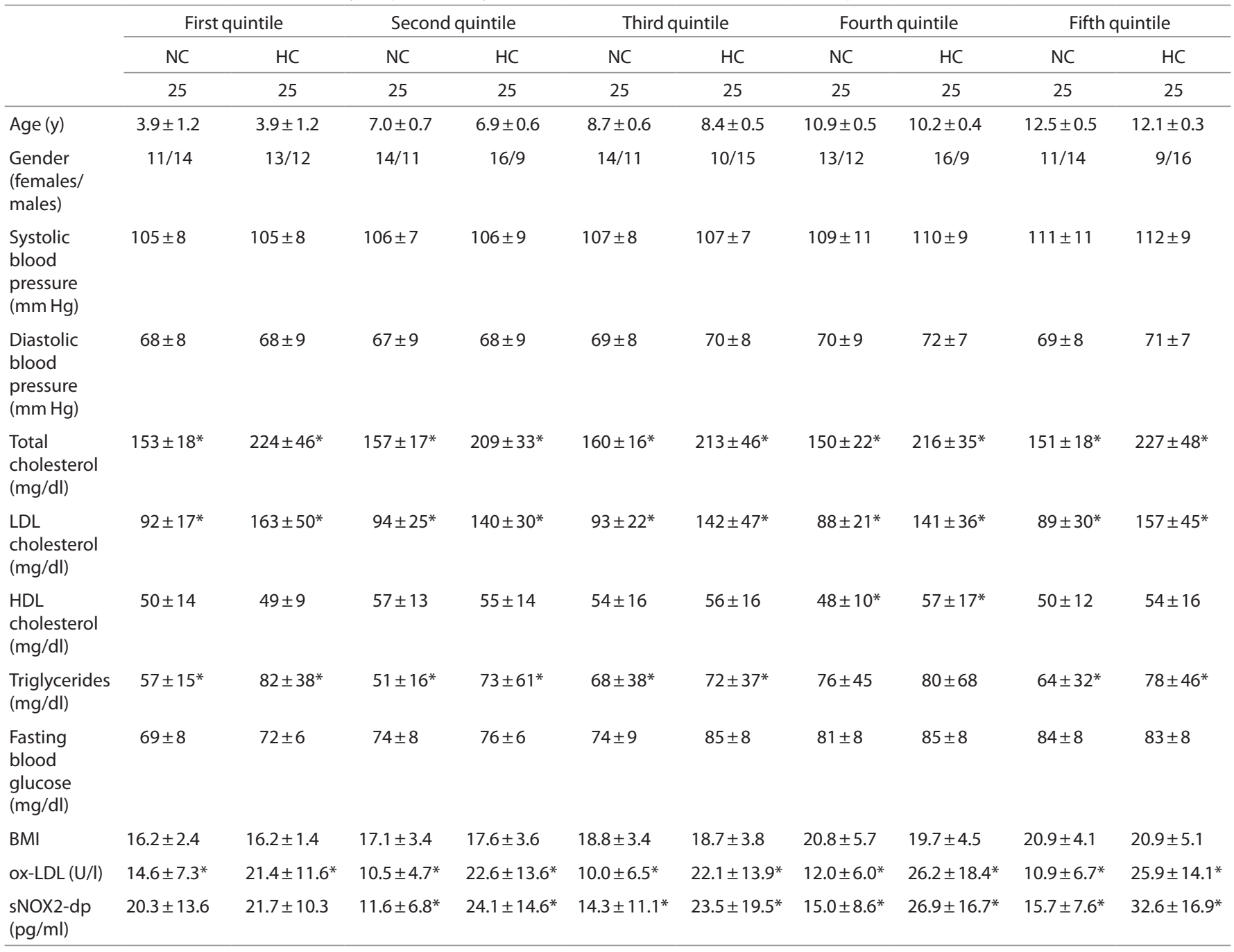

${ }^{*} P<0.05, \mathrm{NC}$ vs. HC

HDL, high-density lipoprotein; LDL, low-density lipoprotein; NC, normocholesterolemic children; ox-LDL, oxidized LDL; sNOX2-dp, soluble NOX2-derived peptide.

those of NC, sNOX2-dp levels were higher in HC from the second to the fifth quintile, suggesting a precocious role for this ROS-generating pathway in enhancing oxidative stress. Furthermore, the significant correlation between sNOX2-dp and ox-LDL suggests a role for this ROS-generating pathway in promoting LDL oxidation. ox-LDL elicits an array of proatherogenic and proinflammatory responses (20) that could contribute to vascular complications in $\mathrm{HC}$.

Together, these results show that hypercholesterolemia in children elicits functional changes that ultimately lead to an early oxidative stress. Such changes are not dependent only on the activation of NOX2 because activation of myeloperoxidase is also involved (10). The mechanism accounting for the upregulation of these two enzymatic pathways is still unknown and deserves further investigation.

This study has some limitations that should be acknowledged. One of the limitations is the small sample size of our population; larger studies need to evaluate the effect of NADPH oxidase in children. Moreover, we cannot exclude that other
NADPH oxidase isoforms, such as NOX1 (8) and NOX4 (8), or other ROS-generating pathways, such as the xanthine oxidase pathway (21), could be potentially implicated in the oxidative stress formation observed in NC and HC.

In conclusion, this study suggests that in $\mathrm{NC}$, NOX2 contributes to generating ox-LDL during the early phase of life and is implicated in the persistent high oxidative stress observed in the prepubertal period of $\mathrm{HC}$.

\section{METHODS}

A total of 250 consecutive subjects, matched for age and gender, including $125 \mathrm{NC}$ and $125 \mathrm{HC}$, were enrolled through a screening program performed in Italian schools for metabolic disease prevention in childhood. In this population, we measured serum ox-LDL, as a marker of oxidative stress, and NOX2 activity as assessed by blood levels of sNOX2-dp. BMI was determined according genderand age-specific growth charts (22). All the children with hypercholesterolemia had a polygenic hypercholesterolemia, classified as the presence of TC $>90$ th age- and gender-specific percentile and without clear family transmission (15). Exclusion criteria for all subjects included the existence of hypothyroidism, renal disease, malignancy, 

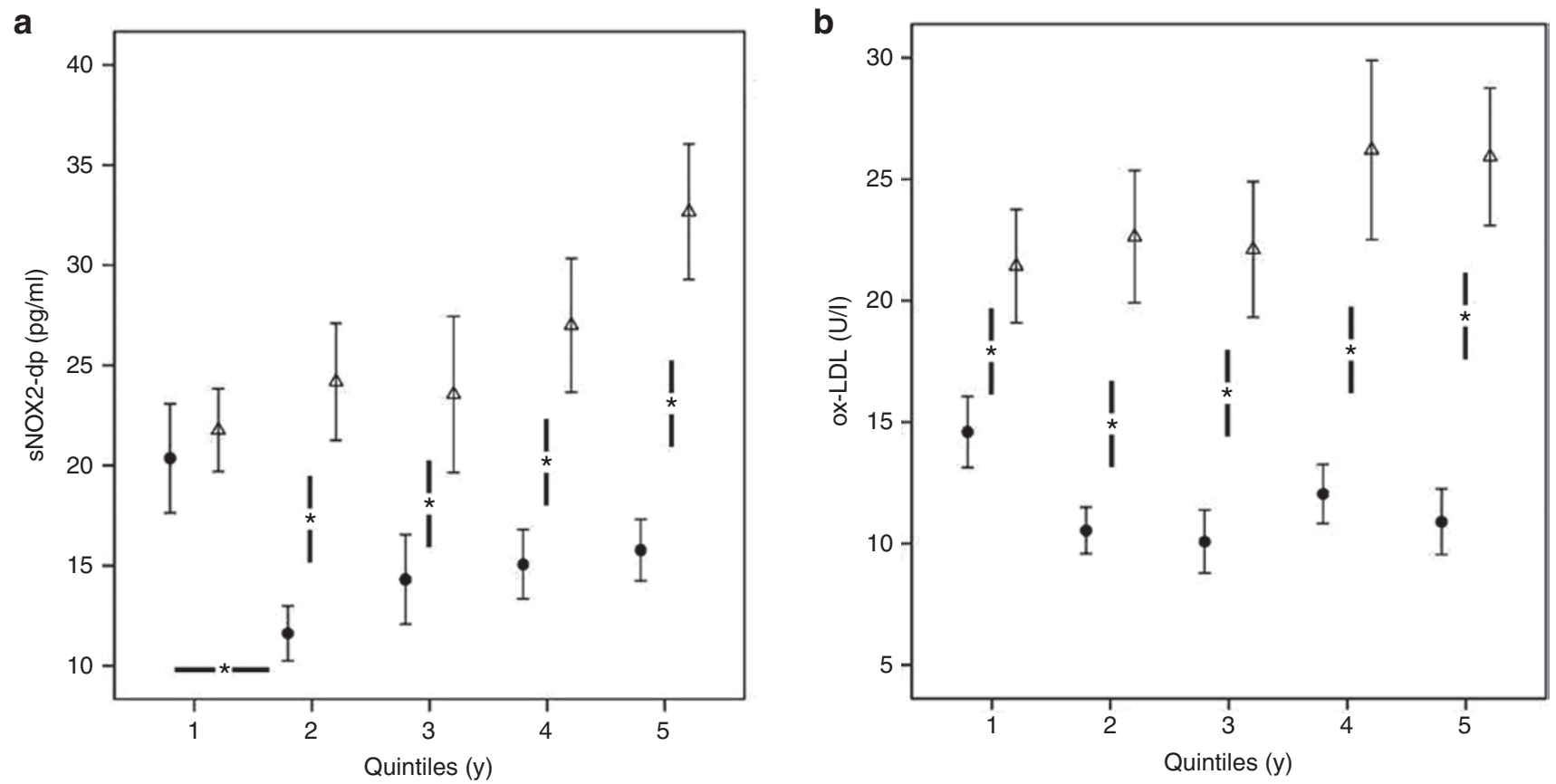

Figure 2. sNOX2-dp and ox-LDL levels according to quintile. (a) sNOX2-dp and (b) ox-LDL values in HC and NC according quintiles. Black circles: NC group; white triangles: HC group. * $P<0.05$. HC, hypercholesterolemic children; NC, normocholesterolemic children; ox-LDL, oxidized low-density lipoprotein; sNOX2-dp, soluble NOX2-derived peptide.

treatment with immunosuppressive drugs, connective tissue disease, and acute illness.

All subjects were enrolled from the same geographic area and followed a typical Mediterranean diet. None of the patients had clinical evidence of cardiovascular disease (as shown by clinical history, physical examination, or electrocardiogram), diabetes mellitus, or hypertension. Patients with hypercholesterolemia had not taken any lipid-lowering agents.

\section{Blood Sampling}

Between 8.00 and $9.00 \mathrm{AM}$, subjects underwent routine biochemical evaluations including fasting TC and glucose levels. After overnight fasting (12 h) and supine rest for at least $10 \mathrm{~min}$, blood samples were collected in vacutainers (Vacutainer Systems, Belliver Industrial Estate, Plymouth England, UK) and centrifuged at 5,000 $\mathrm{g}$ for $10 \mathrm{~min}$ to obtain supernatant, which was stored at $-80^{\circ} \mathrm{C}$ until use.

Serum TC, high-density lipoprotein cholesterol, and triglycerides were measured by an enzymatic colorimetric method. LDL-C levels were calculated according to the Friedwald formula.

Informed written consent was obtained from all subjects. The study conformed to the ethical guidelines of the 1975 Declaration of Helsinki and was approved by the ethical committee of Sapienza University.

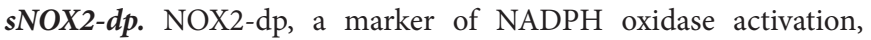
was detected in serum by an enzyme-linked immunosorbent assay method as previously described by Pignatelli et al. (23). The peptide was recognized by the specific monoclonal antibody against the amino acidic sequence (224-268) of the extra membrane portion of NOX2. Values were expressed as $\mathrm{pg} / \mathrm{ml}$; intraassay and interassay coefficients of variation were 5.2 and $6 \%$, respectively.

$\boldsymbol{o x}-\boldsymbol{L D L}$. Serum levels of ox-LDL were measured by commercially available immunoassays (Tema Ricerca, Bologna, Italy). Intraassay and interassay coefficients of variation were 4.0 and $8.3 \%$ for ox-LDL.

\section{Statistical Analyses}

Comparisons between groups were carried out by Student's $t$-test. Data were presented as mean \pm SD unless otherwise indicated.
Comparisons to detect differences among age groups were carried out by ANOVA for multiple measures, with Bonferroni correction for post hoc analysis. Proportions and categorical variables were tested by the $\chi^{2}$ test. The correlation analysis was carried out by Pearson's test. To determine the independent predictors of sNOX2-dp, multiple linear regression analysis was performed using a forward-selection method. Statistical significance was defined at $P<0.05$. Statistical analysis was performed with SPSS 18.0 software for Windows (SPSS, Chicago, IL).

\section{Sample Size Determination}

We computed the minimum sample size with respect to a twotailed one-sample Student's $t$-test, considering (i) a difference for sNOX2-dp to be detected between the healthy subjects and HC as $|\delta| 10 \mathrm{pg} / \mathrm{ml}$, (ii) homogeneous SDs between groups as 16 , and (iii) type I error probability as $\alpha=0.05$ and power $1-\beta=0.90$. This resulted in a minimum sample size of $n=54$ for group.

\section{STATEMENT OF FINANCIAL SUPPORT}

This study was supported by a grant from Sapienza University (Ateneo Federato 2009) (funds to L.L. and F.V.), Rome, Italy.

Disclosure: The authors declared no conflict of interest.

\section{REFERENCES}

1. Tyroler HA. Coronary heart disease epidemiology in the $21^{\text {st }}$ century. Epidemiol Rev 2000;22:7-13.

2. Napoli C, Glass CK, Witztum JL, Deutsch R, D’Armiento FP, Palinski W. Influence of maternal hypercholesterolaemia during pregnancy on progression of early atherosclerotic lesions in childhood: Fate of Early Lesions in Children (FELIC) study. Lancet 1999;354:1234-41.

3. Newman WP 3rd, Freedman DS, Voors AW, et al. Relation of serum lipoprotein levels and systolic blood pressure to early atherosclerosis. The Bogalusa Heart Study. N Engl J Med 1986;314:138-44.

4. Berenson GS, Srinivasan SR, Bao W, Newman WP 3rd, Tracy RE, Wattigney WA. Association between multiple cardiovascular risk factors and atherosclerosis in children and young adults. The Bogalusa Heart Study. N Engl J Med 1998;338:1650-6. 
5. Raitakari OT, Juonala M, Kähönen $\mathrm{M}$, et al. Cardiovascular risk factors in childhood and carotid artery intima-media thickness in adulthood: the Cardiovascular Risk in Young Finns Study. JAMA 2003;290:2277-83.

6. Hagens VE, Ranchor AV, Van Sonderen E, et al.; RACE Study Group. Effect of rate or rhythm control on quality of life in persistent atrial fibrillation. Results from the Rate Control Versus Electrical Cardioversion (RACE) Study. J Am Coll Cardiol 2004;43:241-7.

7. Fuster V, Rydén LE, Cannom DS, et al. 2011 ACCF/AHA/HRS focused updates incorporated into the ACC/AHA/ESC 2006 Guidelines for the management of patients with atrial fibrillation: a report of the American College of Cardiology Foundation/American Heart Association Task Force on Practice Guidelines developed in partnership with the European Society of Cardiology and in collaboration with the European Heart Rhythm Association and the Heart Rhythm Society. J Am Coll Cardiol 2011;57:e101-98.

8. Cave AC, Brewer AC, Narayanapanicker A, et al. NADPH oxidases in cardiovascular health and disease. Antioxid Redox Signal 2006;8:691-728.

9. Wyse DG, Waldo AL, DiMarco JP, et al.; Atrial Fibrillation Follow-up Investigation of Rhythm Management (AFFIRM) Investigators. A comparison of rate control and rhythm control in patients with atrial fibrillation. N Engl J Med 2002;347:1825-33.

10. Chung MK. Randomized trials of rate vs. rhythm control for atrial fibrillation. J Interv Card Electrophysiol 2004;10:Suppl 1:45-53.

11. Corley SD, Epstein AE, DiMarco JP, et al.; AFFIRM Investigators. Relationships between sinus rhythm, treatment, and survival in the Atrial Fibrillation Follow-Up Investigation of Rhythm Management (AFFIRM) Study. Circulation 2004;109:1509-13.

12. Loffredo L. Chronic granulomatous disease. Intern Emerg Med 2011;6:Suppl 1:125-8.
13. Bedard K, Krause KH. The NOX family of ROS-generating NADPH oxidases: physiology and pathophysiology. Physiol Rev 2007;87:245-313.

14. Takac I, Schröder K, Brandes RP. The Nox family of NADPH oxidases: friend or foe of the vascular system? Curr Hypertens Rep 2012;14:70-8.

15. Martino F, Loffredo L, Carnevale R, et al. Oxidative stress is associated with arterial dysfunction and enhanced intima-media thickness in children with hypercholesterolemia: the potential role of nicotinamide-adenine dinucleotide phosphate oxidase. Pediatrics 2008;122:e648-55.

16. Loffredo L, Martino F, Carnevale R, et al. Obesity and hypercholesterolemia are associated with NOX2 generated oxidative stress and arterial dysfunction. J Pediatr 2012;161:1004-9.

17. Weinsier RL, Schutz Y, Bracco D. Reexamination of the relationship of resting metabolic rate to fat-free mass and to the metabolically active components of fat-free mass in humans. Am J Clin Nutr 1992;55:790-4.

18. Van den Bruel A, Bartholomeeusen S, Aertgeerts B, Truyers C, Buntinx F. Serious infections in children: an incidence study in family practice. BMC Fam Pract 2006;7:23.

19. Tsukahara H. Biomarkers for oxidative stress: clinical application in pediatric medicine. Curr Med Chem 2007;14:339-51.

20. Levitan I, Volkov S, Subbaiah PV. Oxidized LDL: diversity, patterns of recognition, and pathophysiology. Antioxid Redox Signal 2010;13:39-75.

21. Dikalov S. Cross talk between mitochondria and NADPH oxidases. Free Radic Biol Med 2011;51:1289-301.

22. Barlow SE; Expert Committee. Expert committee recommendations regarding the prevention, assessment, and treatment of child and adolescent overweight and obesity: summary report. Pediatrics 2007;120:Suppl 4:S164-92.

23. Pignatelli P, Carnevale R, Cangemi R, et al. Atorvastatin inhibits gp91phox circulating levels in patients with hypercholesterolemia. Arterioscler Thromb Vasc Biol 2010;30:360-7. 